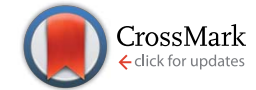

Cite this: RSC Adv., 2017, 7, 14544

Received 24th November 2016 Accepted 24th February 2017

DOI: $10.1039 / c 6 r a 27280 k$

rsc.li/rsc-advances

\section{Fabrication of ultra-smooth and oxide-free molecule-ferromagnetic metal interfaces for applications in molecular electronics under ordinary laboratory conditions $\dagger$}

\begin{abstract}
Karuppannan Senthil kumar, ${ }^{a}$ Li Jiang $^{a}$ and Christian A. Nijhuis ${ }^{\star a b}$
Self-assembled monolayers of alkanethiolates on ferromagnetic metal surfaces have potential applications in molecular spintronics, but the fabrication of such structures is complicated by unwanted oxidation of the ferromagnetic metal. This paper describes the fabrication of ultra-smooth oxide-free $\mathrm{Ni}$ surfaces via template-stripping which are protected by SAMs of $\mathrm{S}\left(\mathrm{CH}_{2}\right)_{n-1} \mathrm{CH}_{3}$ that are stable for 1 day in ambient environment. Our method does not require ultra-high vacuum conditions, glove-box techniques, or (redox) cleaning of the Ni surface, but can be readily applied under ordinary laboratory conditions. Passivation of the $\mathrm{Si} / \mathrm{SiO}_{2}$ template with a layer of FOTS $(1 \mathrm{H}, 1 \mathrm{H}, 2 \mathrm{H}, 2 \mathrm{H}$-perfluorooctyltrichlorosilane) reduced the $\mathrm{Ni}$ template interaction sufficiently enabling successful template-stripping. The $\mathrm{Ni}^{\mathrm{TS}}$-SAM interfaces were characterized by X-ray photoelectron spectroscopy (XPS) and atomic force microscopy (AFM). We found that the surfaces were ultra-flat with a root mean square surface roughness of $0.15 \pm 0.05 \mathrm{~nm}$ over $1.0 \times$ $1.0 \mu \mathrm{m}^{2}$ and that they were stable against oxidation for 1 day in air at room temperature. These SAMs on $\mathrm{Ni}$ were incorporated in SAM-based tunneling junctions of the form $\mathrm{Ni}^{\mathrm{TS}}-\mathrm{SC}_{n} / / \mathrm{GaO}_{x} / \mathrm{EGaln}$ to study the tunneling rate across the SAMs. The tunneling rate is highly sensitive to defects in the SAMs or the presences of oxides. We found that the charge transport properties across these junctions were indistinguishable from those junctions with formed on $\mathrm{Au}^{\mathrm{TS}}$ and $\mathrm{Ag}^{\mathrm{TS}}$ substrates from which we conclude that our method yields high quality $\mathrm{Ni}^{\mathrm{TS}}$-SAM interfaces suitable for applications in molecular electronics.
\end{abstract}

\section{Introduction}

Self-assembled monolayers (SAMs) of thiol-functionalized molecules on ferromagnetic metal (FM) surfaces are interesting for possible applications in molecular, ${ }^{1-5}$ and biomolecular-spintronics. ${ }^{6-8}$ Such studies are challenging because the formation of metal oxides has to be minimized to ensure efficient spin-dependent charge transport ${ }^{1-4}$ and thus there is a need to have easy access to high quality, oxide free, and defect free FM electrodes. Template-stripping (TS) is a well-known technique to generate ultra-smooth and clean metal surfaces on demand. ${ }^{9-12}$ Often, a clean and ultra-smooth silicon (Si) surface with its native layer of $\mathrm{SiO}_{2}$ is used as the template onto which a thin metal film is deposited. Next, a support, e.g., a glass surface, is glued against the metal and the support/glue/ metal stack is stripped off the template to expose the metal

${ }^{a}$ Department of Chemistry, National University of Singapore, 3 Science Drive 3, Singapore 117543, Singapore

${ }^{b}$ Centre for Advanced $2 D$ Materials and Graphene Research Centre, National University of Singapore, 6 Science Drive 2, Singapore 117546, Singapore. E-mail: christian. nijhuis@nus.edu.sg; Tel: +65 65162667

$\dagger$ Electronic supplementary information (ESI) available: Experimental details. See DOI: $10.1039 / \mathrm{c} 6 \mathrm{ra} 27280 \mathrm{k}$ surface that had been in contact with the template when needed. Since the metal surface was in contact with the template it is protected from the ambient environment and the template serves in this regard as a protective barrier which avoids contamination and oxidation of the metal surface. Although this method works well for inert metals such as Ag, $\mathrm{Au}, \mathrm{Pd}$, or Pt, reactive metals tend to interact too strongly with the template preventing successful template-stripping.

Here, we report a method to fabricate ultra-smooth and oxide free template-stripped nickel $\left(\mathrm{Ni}^{\mathrm{TS}}\right)$ surfaces that can be obtained under ordinary laboratory conditions. This method makes it possible to strip off $\mathrm{Ni}$ from the $\mathrm{Si} / \mathrm{SiO}_{2}$ template via passivation of the template with a monolayer with a low surface energy. We characterized the SAM and $\mathrm{Ni}^{\mathrm{TS}}$ surfaces with X-ray photoelectron spectroscopy (XPS) to confirm successful fabrication of oxide free FM-SAM interfaces. Our method does not rely on any ultra-high vacuum (UHV) environments and/or electrochemical reduction/cleaning processes. ${ }^{13-16}$ Prior to template-stripping, the Ni substrates can be stored in ambient conditions for a period of time of 4-6 months. To demonstrate their usefulness, we prepared good quality molecular tunneling junctions of the form $\mathrm{Ni}^{\mathrm{TS}}-\mathrm{SC}_{n} / / \mathrm{GaO}_{x} / \mathrm{EGaIn}$, where, the $\mathrm{SC}_{n}$ is a SAM of $n$-alkanethiolates of the form of $\mathrm{S}\left(\mathrm{CH}_{2}\right)_{n-1} \mathrm{CH}_{3}$ with 
$n=10-18$. The $\mathrm{GaO}_{x} /$ EGaIn top-electrodes have been wellcharacterized and EGaIn stands for eutectic mixture of $\mathrm{Ga}$ and In $\left(\mathrm{mp}=15.7^{\circ} \mathrm{C}\right)$ which has a self-limiting surface layer of conductive, $0.7 \mathrm{~nm}$ thick $\mathrm{GaO}_{x}$ (predominantly consisting of $\left.\mathrm{Ga}_{2} \mathrm{O}_{3}\right) \cdot{ }^{17,18}$ The electrical properties of the tunnel junctions are given by eqn (1), in which tunneling processes are characterized by the exponential decay of the current density $J$ (in $\mathrm{A} \mathrm{cm}^{-2}$ ) as a function of the tunneling distance $d$ (in $\mathrm{nm}$ ).

$$
J=J_{0, \mathrm{~V}} \mathrm{e}^{-\beta d}
$$

where the $\beta\left(\mathrm{nC}^{-1}\right)$ is the tunneling decay coefficient and $J_{0}\left(\mathrm{~A} \mathrm{~cm}^{-2}\right)$ is the pre-exponential factor for a predefined applied bias. $^{19-22}$ The results show that the SAMs form good quality tunneling barriers on $\mathrm{Ni}^{\mathrm{TS}}$ with $\beta=1.01 \pm 0.03 \mathrm{nC}^{-1}$ and $\log _{10}\left|J_{0}\right|=2.3 \pm 0.2 \mathrm{~A} \mathrm{~cm}^{-2}$; these values are well within the range of consensus values. ${ }^{20-23}$ These junctions also show good yields $(>90 \%)$ in non-shorting junctions and high precision of data with log-standard deviations of 0.3-0.5 similar to junctions with Ag or Au bottom electrodes. ${ }^{19-23}$

The self-assembly of alkyl/aryl thiols on FM surfaces, such as nickel (Ni), ${ }^{6,13-16,24}$ cobalt (Co), ${ }^{14,15,24,25}$ and iron (Fe), ${ }^{14}$ are challenging due to the presence of the native oxide which hampers the formation of the metal-thiolate bonds. Therefore, SAMs are immobilized on FM surfaces via this native oxide layer using carboxylate, phosphonate, or silane, anchoring groups. ${ }^{26-29}$ The presence of a native oxide layer, however, often forms an antiferromagnetic (AFM) passivation layer which hinders the reorientation of ferromagnetic moment of the FM layer by exchange coupling lowering the performance of, for example, spin valves. ${ }^{30-32}$ Thus, to develop fabrication methods to obtain oxide free molecular-FM interfaces are important for molecular spintronics.

Fabrication methods based on highly controlled ultra-high vacuum (UHV) environments or in situ electrochemical control are available..$^{4,53-36}$ Most electrochemical techniques are based on a two-step procedure involving removal of the native oxide by electrochemical reduction, followed by immediate substrate immersion in alkanethiol containing ethanolic solutions. During transfer of the oxide free Ni surface, metal-oxides can form as the substrates are exposed to air. For example, Bengió et $a l .^{34}$ reported the fabrication of $n$-alkanethiolate SAMs on $\mathrm{Ni}(111)$ and polycrystalline Ni surfaces by the electrochemical reduction method in which the electrochemical reduction was performed under either acidic or basic aqueous conditions, while the SAM formation was carried out either in situ or by pulling the substrate through a top layer of neat thiol. Instead of using two separate steps and acidic or basic aqueous electrolyte, Fontanesi et $a .^{35}$ performed electrochemical removal of the oxide layer with the thiol precursor present in the electrolyte (1:0.8, ethanol : water) and reported good quality SAMs. Hoertz et al. ${ }^{14}$ used three different methods for SAM formation on Ni, Co and Fe substrates, (i) glovebox techniques (freshly evaporated metal transfer to a solution of the SAM precursor without exposure to air), (ii) electrochemical reduction inside a glovebox (oxide removal by electrochemical reduction and SAM formation carried out inside the glovebox), and (iii) deposition of metal via direct metal evaporation followed by a brief exposure in air before immersion into a solution with the SAM precursor. The stability of hexadecanethiolate SAM prepared by method (i) and (ii) on FMs was examined by XPS, the sulfur atom oxidized to a $\mathrm{S}=\mathrm{O}$ containing species (e.g., sulfonate, sulfinate, sulfone) within 2 hours of exposure to the ambient atmosphere confirmed by the appearance of $S 2 p$ peak at $\sim 169 \mathrm{eV}$. After five days of exposure the sample contained predominantly oxidized sulfur atoms. The oxidation of $\mathrm{Ni}$ surfaces slows down by the presence of SAMs under atmospheric conditions. Hoertz et al. ${ }^{14}$ reported, alkanethiolate SAM on Ni surface slowed down the formation of the Ni oxide by 1-2 hours using X-ray photoelectron spectroscopy (XPS). As a group, these studies show that storing of FM surfaces is challenging.

Defects in molecular tunneling junctions, such as pinholes, need to be minimized to ensure good quality tunnelling junctions and therefore the surface roughness of the FM surface needs to be kept at a minimum..$^{37-42}$ Substrates used for preparation of SAMs on $\mathrm{Ni}$ by electrochemical methods have typically a root mean square (rms) surface roughness of $2.5 \mathrm{~nm}$ over an area of $2 \times 2 \mu \mathrm{m}^{2}$ which is much higher than the molecular dimensions of the SAMs they support. ${ }^{35}$ In addition, electrochemical etching increases the rms surface roughness of, for example, Ni and $\mathrm{Co}$, by about $\sim 40 \% .{ }^{14}$ Although the roughness is usually reported in terms of rms surface roughness, the topography can also be more comprehensively analyzed to include, grain size, width of the grain boundary, presence of pinholes, and whether the grains are located in the same plane or not. The so-called bearing volume $\left(\mathrm{BV}, \mathrm{nm}^{3}\right)$ of the surface topography includes all of these parameters but a BV analysis has been only occasionally reported. ${ }^{11}$ In this context, TS seems to be an attractive choice to generate FM surfaces because TS produces ultra-flat surfaces, the template provides protection from the atmosphere, and clean metal surfaces are available on demand, and therefore we developed a TS procedure to generate flat and clean Ni surfaces on-demand.

\section{Experimental details}

We purchased nickel (Ni) with purity of 99.999\% from Super Conductor Material, Inc (USA) and silicon wafers (100, p-type, $500 \pm 25 \mu \mathrm{m}$ ) with one side polished from University Wafers (USA). The $1 H, 1 H, 2 H, 2 H$-perfluorooctyltrichlorosilane (FOTS) and alkanethiols $\mathrm{SC}_{n}$ (with $n=10,12,14,16$ and 18) were purchased from Sigma-Aldrich with a purity of $98 \%$. We purified the alkanethiols before use as reported previously. ${ }^{39}$ The $n$ alkanethiol with $n=6$ was purified by distillation under vacuum. The $n$-alkanethiols with $n=10,12,14,16$ and 18 were purified by column chromatography over silica gel with hexane as an eluent and then recrystallised from ethanol under an atmosphere of $\mathrm{N}_{2}$ followed by quick filtration. The galliumindium eutectic $(75.5 \% \mathrm{Ga}$ and $24.5 \%$ In by weight) was purchased from Sigma-Aldrich. The solvents were AR grade.

\section{Fabrication of $\mathrm{Ni}^{\mathrm{TS}}$ substrate}

$300 \mathrm{~nm}$ Ni was evaporated on $1 H, 1 H, 2 H, 2 H$-perfluorooctyltrichlorosilane (FOTS) functionalized $\operatorname{Si}(\langle 100\rangle)$ substrate by 
e-beam evaporation. Before $\mathrm{Ni}$ evaporation, the silicon substrate was exposed in FOTS for $30 \mathrm{~min}$ at $1 \mathrm{mbar}$ in vacuum desiccators. $300 \mathrm{~nm}$ layer of $\mathrm{Ni}$ was evaporated at a rate of $0.1 \AA$ $\mathrm{s}^{-1}$ monitored by a quartz crystal thickness controller. We cleaned the glass slides by ultra-sonication in ethanol solution, followed by washing with ethanol, the slides were blown to dryness in a stream on $\mathrm{N}_{2}$ gas. Before we glued the glass on the nickel surfaces with optical adhesive (Norland, No. 61), the glass slides were cleaned by a plasma of air for $5 \mathrm{~min}$ at a pressure of 5 mbar. The optical adhesive was cured for 2 hours under UVlight irradiation following previously reported methods. ${ }^{9,39}$ In order to prevent nickel oxidation template stripping was carried out in a $\mathrm{N}_{2}$ flow hood.

\section{Formation of SAMs on $\mathrm{Ni}^{\mathrm{TS}}$}

The SAMs of alkanethiolates ( $\mathrm{SC}_{n}$ with $n=10,12,14,16$ and 18) were prepared by immersing freshly template-stripped $\mathrm{Ni}^{\mathrm{TS}}$ from the silicon wafer inside the $\mathrm{N}_{2}$ flow hood into $3 \mathrm{mM}$ ethanolic solutions of the alkanethiolate molecule. The SAMs were formed overnight under a $\mathrm{N}_{2}$ atmosphere (Fig. 1) using previously reported methods. ${ }^{43}$ Then the SAM-modified substrates were rinsed with ethanol and blown to dryness in a stream of $\mathrm{N}_{2}$ gas.

\section{Monolayer characterization}

The surface chemical composition of the SAMs chemisorbed on $\mathrm{Ni}^{\mathrm{TS}}$ substrate was characterized by XPS using well-established procedures. ${ }^{44}$ The photoemission spectra were measured using VG ESCA lab-220i XL XPS. The system was equipped with a monochromatic $\mathrm{Al} \mathrm{K} \alpha \mathrm{X}$-ray source with a photon energy of $1486.6 \mathrm{eV}$ at $15 \mathrm{kV}$. The high resolution spectra were collected at a pass energy of $20 \mathrm{eV}$, with $0.1 \mathrm{eV}$ steps, at a $45^{\circ}$ take-off angle. The binding energies were corrected against the $\mathrm{C}$ 1s energy of $285.0 \mathrm{eV}$. The collected XPS high resolution spectra were analysed using XPS Peak fit 4.1 software using Voigt functions (30\% Lorentzian and a 70\% Gaussian). The background from each spectrum was subtracted using Shirley-type back grounds to remove most of the extrinsic loss structure. The SAM thickness and tilt angle were measured using angle resolved photoelectron

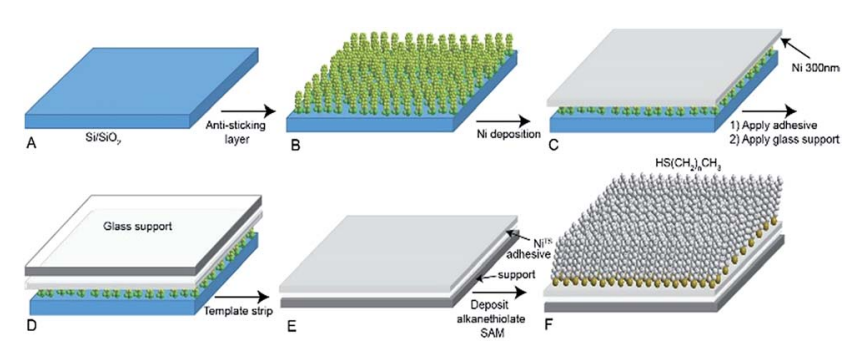

Fig. 1 Schematic illustration of the fabrication process of templatestripped $\mathrm{Ni}\left(\mathrm{Ni}^{\mathrm{TS}}\right)$ coated with a SAM. (A) On a clean $\mathrm{Si} / \mathrm{SiO}_{2}$ surface (B) FOTS was deposited followed by (C) deposition of $300 \mathrm{~nm}$ Ni. (D) Glass supports were glued on the Ni surface using photocurable optical adhesive. (E) After curing of the adhesive, the metal film around the glass support was cut out using a razor blade. Next, the nickel film, with its glass support, was lifted off to expose an ultra-flat $\mathrm{Ni}$ film (inside of a $\mathrm{N}_{2}$ flow hood). (F) The $\mathrm{Ni}^{\mathrm{TS}}$ surface was then immediately transferred into a solution of $n$-alkanethiolates. spectroscopy (AR-XPS). We used synchrotron-based photoelectron spectroscopy to perform the angle resolved XPS measurements at the SINS (Surface, Interface and Nanostructure Science) beamline of Singapore Synchrotron Light Source (SSLS). The measurements were carried out at room temperature under ultra-high vacuum with a base pressure of $10^{-10}$ mbar. We recorded S 2p spectra at different angles of from $0^{\circ}$ to $90^{\circ}$ to measure surface coverage and tilt angle of SAMs on $\mathrm{Ni}^{\mathrm{TS}}$ surface.

\section{Ultraviolet photoelectron spectroscopy (UPS)}

We determined the work function of the $\mathrm{Ni}^{\mathrm{TS}}$ surfaces with and without SAMs with UPS at the SINS beam-line. ${ }^{44}$ The top of the valence band and work function $\Phi$ values were determined by linear extrapolation of the lower binding energy side of the HOMO peak and secondary electron cut-off to the base line.

\section{Atomic force microscopy (AFM)}

AFM images were performed in the tapping mode, using Bruker dimension Fastscan (FASTSCAN-A, resonant frequency: 1.4 $\mathrm{MHz}$, force constant: $18 \mathrm{~N} \mathrm{~m}^{-1}$ ). We used nanoscope analysis (version 1.4) software to analyse the AFM images.

\section{Junction fabrication and data collection}

The junction fabrication and data collection has been reported before. ${ }^{44}$ Here, we give only a brief description. We used coneshaped EGaIn tips to approach the Ni-SAM substrates and form a junction with a diameter of 20-30 $\mu \mathrm{m}$. For each SAM, we fabricated junctions on four to five different samples and collected five to six junctions on each sample. For each junction, we first carried out 5 scans to stabilize the junction and then collected 20 traces (except for shorts), which were all used for data analysis. We defined a short when the value of $J$ exceeded $10^{2} \mathrm{~A} \mathrm{~cm}^{-2}$ (the compliance value of $J$ of our instrument) during scanning (including the first 5 scans). The yield of non-shorting junctions is defined as the number of stable junctions divided by the total number of junctions. We followed the procedure for statistical analysis of the junction data as reported before. ${ }^{2}$ Briefly, we plotted the value of $\log _{10}|J|$ at a given bias voltage in histograms and fitted Gaussians to these histograms to obtain the log-standard deviation and log-mean of the value of $J$. We used this procedure for all the applied biases to construct the log-average $J(V)$ curves.

\section{Results and discussion}

Fabrication of $\mathrm{Ni}^{\mathrm{TS}}$ substrates

Fig. 1 shows schematically the procedure for the preparation the $\mathrm{Ni}^{\mathrm{TS}}$ substrates and formation of alkanethiolate SAMs of $\mathrm{S}\left(\mathrm{CH}_{2}\right)_{n-1} \mathrm{CH}_{3}$ (in short $\mathrm{SC}_{n}$ ) on these $\mathrm{Ni}^{\mathrm{TS}}$ surfaces. As a template, we tried to use a $\mathrm{Si}$ wafer with its native $\mathrm{SiO}_{2}$ layer $\left(\mathrm{Si} / \mathrm{SiO}_{2}\right)$ or an oxide free $\mathrm{Si}$ template. We found that the nickel cannot be stripped off successfully because $\mathrm{Ni}$ interacted too strongly with these templates. To be able to peel off the nickel layer from the template, the Ni-template interaction has to be minimized. Besides, physisorbed and/or chemisorbed water on the template plays a role in the formation of nickel oxides. ${ }^{45}$ To 
minimize the formation of $\mathrm{NiO}$ bonds with the $\mathrm{SiO}_{2}$ surface and to increase the hydrophobicity of the template, we formed a monolayer of $1 \mathrm{H}, 1 \mathrm{H}, 2 \mathrm{H}, 2 \mathrm{H}$-perfluorooctyltrichlorosilane (FOTS) on the $\mathrm{Si} / \mathrm{SiO}_{2}$ template via gas phase deposition for 30 min at $5 \times 10^{-2}$ mbar in a vacuum desiccator (Fig. 1B). We found that this approach effectively reduced the Ni-template interaction enabling us to obtain $\mathrm{Ni}^{\mathrm{TS}}$ in high yields. On the $\mathrm{Si} / \mathrm{SiO}_{2}-\mathrm{FOTS}$ template a layer of $300 \mathrm{~nm}$ thick $\mathrm{Ni}$ was deposited (Fig. 1C). Next, glass substrates $\left(1 \times 1 \mathrm{~cm}^{2}\right)$ were glued against the Ni layer using an epoxy glue (Norland No. 61) which was cured under UV-light irradiation following previously reported procedures (Fig. 1D). ${ }^{9,11,46}$ Finally, we stripped of the glass/AO/Ni stacks from the $\mathrm{Si} / \mathrm{SiO}_{2}-\mathrm{FOTS}$ template in a $\mathrm{N}_{2}$ flow hood to yield oxide-free $\mathrm{Ni}^{\mathrm{TS}}$ surfaces that had been in contact with the template (Fig. 1E). These freshly prepared $\mathrm{Ni}^{\mathrm{TS}}$ surfaces were immersed immediately (within a few seconds) into ethanolic solutions of $\mathrm{HSC}_{n}$ to form SAMs overnight under a $\mathrm{N}_{2}$ atmosphere (Fig. 1F). The samples were washed with ethanol and then dried in a stream of $\mathrm{N}_{2}$.

\section{Topography of the $\mathrm{Ni}^{\mathrm{TS}}$ surface}

We characterized the topography of the $\mathrm{Ni}^{\mathrm{TS}}$ surfaces with tapping mode AFM and compared the results against $\mathrm{Ni}$ surfaces obtained by direct evaporation of $\mathrm{Ni}$ on $\mathrm{Si} / \mathrm{SiO}_{2}$ via ebeam evaporation $\left(\mathrm{Ni}^{\mathrm{DE}}\right)$. Fig. 2 shows the AFM images of an as-deposited nickel surface $\mathrm{Ni}^{\mathrm{DE}}(300 \mathrm{~nm})$ on $\mathrm{SiO}_{2} / \mathrm{Si}$ and $\mathrm{Ni}^{\mathrm{TS}}$. As expected, the rms surface roughness of the $\mathrm{Ni}^{\mathrm{TS}}$ surfaces of $0.15 \pm 0.05 \mathrm{~nm}$ over $1.0 \times 1.0 \mu \mathrm{m}^{2}$ are substantially smaller than those of $\mathrm{Ni}^{\mathrm{DE}}$ surfaces of $2.0 \pm 0.1 \mathrm{~nm}$ measured over the same area. However, the topography contains more information rather than the surface roughness, such as the size of grains, the width of the grain boundaries, or the presence of pinholes. Based on previous studies, we found that exposed surface grain boundaries are the major source of defects in SAM-based tunneling junctions. ${ }^{22,39,47}$ The exposed grain boundary area depends on the rms surface roughness (which essentially tells us whether the grains are in the same plain), grain boundary width, and size of the grains. For instance, a surface with large grains and small grain boundary widths has a smaller surface fraction of exposed grain boundaries than a surface with small grains and large grain boundary widths. Therefore, we used the bearing volume (BV), as defined in eqn (2), to determine the quality of metal surfaces

$$
\mathrm{BV}=N_{\mathrm{gr}} \times A_{\mathrm{gb}} \times \mathrm{rms}
$$
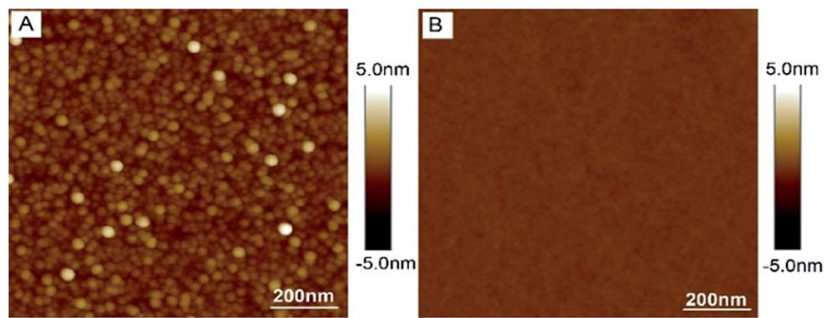

Fig. 2 Atomic force microscopy (AFM) image of (A) as deposited $\mathrm{Ni}^{\mathrm{DE}}$ $(300 \mathrm{~nm})$ on $\mathrm{SiO}_{2} / \mathrm{Si}$-substrate and $(\mathrm{B}) \mathrm{Ni}^{\mathrm{TS}}$. where $N_{\mathrm{gr}}$ is the relative number of grains normalized by the largest grain size (where the value of $N_{\mathrm{gr}}$ is 1 for the surfaces with the largest grain size and the values of $N_{\mathrm{gr}}$ for other surfaces are the ratios of the $\left.A_{\mathrm{gr}}^{\text {largest }} / A_{\mathrm{gr}}^{\text {other }}\right), A_{\mathrm{gb}}$ is the area of grain boundaries, and rms is the root mean square surface roughness (see ESI $\dagger$ and ref. 39 for the details). Generally, low $\mathrm{BV}$ values indicate smooth surfaces with a small fraction of exposed grain boundaries, while high BV values imply defective surfaces with a large surface fraction of exposed grain boundaries. The $\mathrm{BV}$ value of $\mathrm{Ni}^{\mathrm{TS}}$ surface is around $2 \times 10^{5} \mathrm{~nm}^{3}$ and that of $\mathrm{Ni}^{\mathrm{DE}}$ is one order of magnitude higher $\left(2 \times 10^{6} \mathrm{~nm}^{3}\right)$, which indicates the quality of $\mathrm{Ni}^{\mathrm{TS}}$ is better than that of $\mathrm{Ni}^{\mathrm{DE}}$ surface. The $\mathrm{BV}$ value of $\mathrm{Ni}^{\mathrm{TS}}$ surface is similar to the high quality $\mathrm{Au}^{\mathrm{TS}}$ and $\mathrm{Ag}^{\mathrm{TS}}$ surfaces reported before which have been used successfully to fabricate high quality SAM-based junctions. ${ }^{9,11,47}$

\section{XPS characterization of $\mathrm{Ni}^{\mathrm{TS}}$ and $\mathrm{Ni}^{\mathrm{TS}}-\mathrm{SAM}$}

We measured XPS spectra of $\mathrm{F} 1 \mathrm{~s}$ to determine whether the $\mathrm{Ni}^{\mathrm{TS}}$ surface contains any residues of the FOTS passivation layer. It is well-known that the C-F bond is prone to radiation damage, ${ }^{48,49}$ therefore we recorded the high resolution $\mathrm{F}$ 1s spectra before the survey scans and the other XPS measurements. Fig. S1† shows the F 1s spectrum and that no F 1s signal could detected which confirms that the $\mathrm{Ni}^{\mathrm{TS}}$ surface is clean and does not contain residues (within the detection limit of our XPS) of the FOTS anti-sticking layer. We further analyzed the $\mathrm{Ni}^{\mathrm{TS}}$ and $\mathrm{Ni}^{\mathrm{TS}}$ SAM surfaces by XPS to verify the chemical composition of the system with the specific aim to establish whether nickel oxide (NiO) formation was successfully prevented. Fig. 3 shows the oxygen (O 1s), nickel (Ni 2p), sulfur (S 2p), and carbon (C 1s), spectra of the $\mathrm{Ni}^{\mathrm{TS}}$ and $\mathrm{Ni}^{\mathrm{TS}}-\mathrm{SAM}$ surfaces and the corresponding binding energies are summarized in Table 1. No clear signals were observed in the $\mathrm{O}$ 1s spectra (Fig. 3A) obtained from the $\mathrm{Ni}^{\mathrm{TS}}-\mathrm{SAM}$ substrates which confirm that no oxides

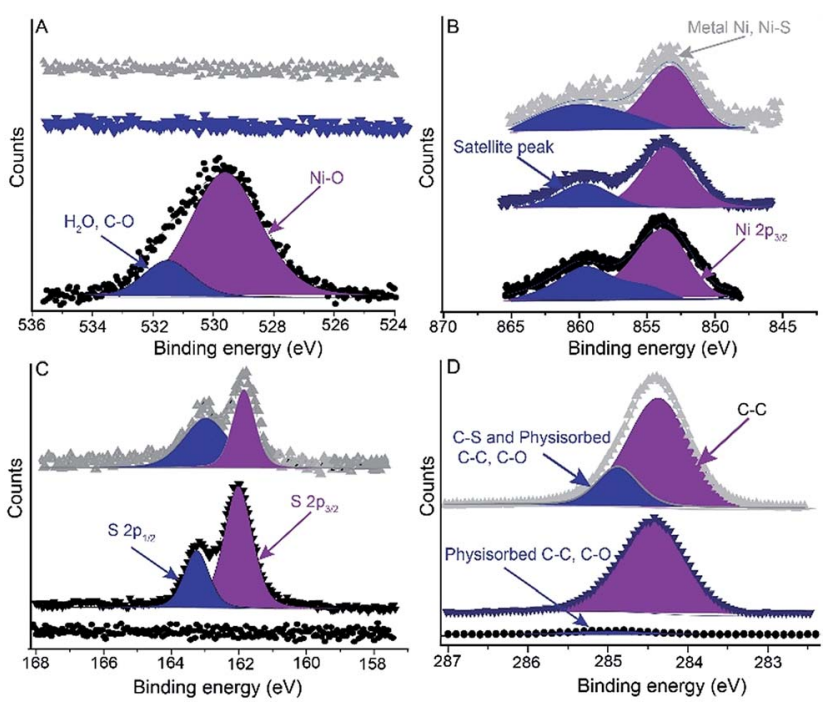

Fig. 3 XPS spectra of (A) O 1s, (B) Ni 2p, (C) S 2p and (D) C 1s of the (O) bare $\mathrm{Ni}^{\mathrm{TS}},(\boldsymbol{\nabla}) \mathrm{Ni}^{\mathrm{TS}}-\mathrm{C}_{6}$ and $(\boldsymbol{\Delta}) \mathrm{Ni}^{\mathrm{TS}}-\mathrm{C}_{14}$ substrates. 
Table 1 Measured binding energies of the major core lines of SAMs on $\mathrm{Ni}^{\mathrm{TS}}$ under atmospheric conditions at different time intervals

\begin{tabular}{lllll}
\hline Sample & $\mathrm{C} 1 \mathrm{~s}(\mathrm{eV})$ & $\mathrm{O} 1 \mathrm{~s}(\mathrm{eV})$ & $\mathrm{Ni} 2 \mathrm{p}^{a}(\mathrm{eV})$ & $\mathrm{S} \mathrm{2p}(\mathrm{eV})$ \\
\hline $\mathrm{Ni}^{\mathrm{TS}}$ & 285.0 & $529.5,531.6$ & $854.4,860.0$ & - \\
$\mathrm{Ni}^{\mathrm{TS}}-\mathrm{C}_{6}$ & 284.4 & - & $853.9,860.3$ & $162.0,163.2$ \\
$\mathrm{Ni}^{\mathrm{TS}}-\mathrm{C}_{14}$ & $284.5,284.8$ & - & $853.9,860.3$ & $161.4,162.6$ \\
$\mathrm{Ni}^{\mathrm{TS}}-\mathrm{C}_{14}(24 \mathrm{~h})$ & $284.5,284.8$ & $529.8,530.8$ & $854.0,860.3$ & $161.3,162.4$ \\
$\mathrm{Ni}^{\mathrm{TS}}-\mathrm{C}_{14}(48 \mathrm{~h})$ & $284.5,284.9$ & & $854.3,860.2$ & 163.65 \\
${ }^{a}$ Binding energy of Ni 2p & & & & - \\
\end{tabular}

were present. In contrast, the bare $\mathrm{Ni}^{\mathrm{TS}}$ substrates clearly show an $\mathrm{O} 1 \mathrm{~s}$ signal at $529.5 \mathrm{eV}$ corresponding to NiO. The $\mathrm{O} 1 \mathrm{~s}$ spectra are in agreement with the $\mathrm{Ni} 2 \mathrm{p}_{3 / 2}$ spectra (Fig. 3B) which show that the overlapping metal-oxide $(\mathrm{Ni}-\mathrm{O})$ at $854.4 \mathrm{eV}$ is absent for the $\mathrm{Ni}^{\mathrm{TS}}-\mathrm{SAM}$ samples and only a $\mathrm{Ni}-\mathrm{S}$ peak at $853.9 \mathrm{eV}$ can be observed. From these spectra we conclude, that within the detection limits of XPS, that a SAM on oxide free $\mathrm{Ni}^{\mathrm{TS}}$ can be formed. We note, however, that the satellite peak in Fig. 3B is not completely symmetrical which could be caused by a small amount of nickel oxides.

To confirm the presence of the SAM, we measured the $\mathrm{S} 2 \mathrm{p}$ and $\mathrm{C}$ 1s spectra for from the $\mathrm{Ni}^{\mathrm{TS}}$-SAM substrates. The $\mathrm{S} 2 \mathrm{p}$ spectrum is dominated by a doublet with the main component at $161.4 \mathrm{eV}\left(2 \mathrm{p}_{3 / 2}\right)$ and the minor component at $162.6 \mathrm{eV}\left(2 \mathrm{p}_{1 / 2}\right)$. These values are typical for metal-thiolates and these data confirm the presence of the SAM. The C 1s spectra are dominated by a peak at $284.6 \mathrm{eV}$ which is associate with the alkyl chain of the SAM. The weak signal at $285.0 \mathrm{eV}$ for bare $\mathrm{Ni}^{\mathrm{TS}}$ is due to physisorbed carbon contaminants on the $\mathrm{Ni}^{\mathrm{TS}}$ surface. Although the $\mathrm{SC}_{6} \mathrm{SAM}$ was dominated by one peak, the $\mathrm{SC}_{14}$ SAM sample had an additional weak signal due to carbon contamination which likely physisorbed during transfer into the XPS chamber. From these data we conclude that both SAMs effectively prevented the formation of nickel-oxides.

\section{Monolayer thickness, tilt angle- and work function of $\mathrm{Ni}^{\mathrm{TS}}$ SAM}

We used angle resolved XPS to determine the SAM thickness, $d_{\mathrm{SAM}}$ in $\mathrm{nm}$, on the $\mathrm{Ni}^{\mathrm{TS}}$ following previously reported procedures. ${ }^{39}$ We recorded $\mathrm{S} 2 \mathrm{p}$ spectra at five different angles ranging from $20^{\circ}$ to $90^{\circ}$ (Fig. $4 \mathrm{~A}$; see ESI $\dagger$ ). The value of $d_{\mathrm{SAM}}$ for

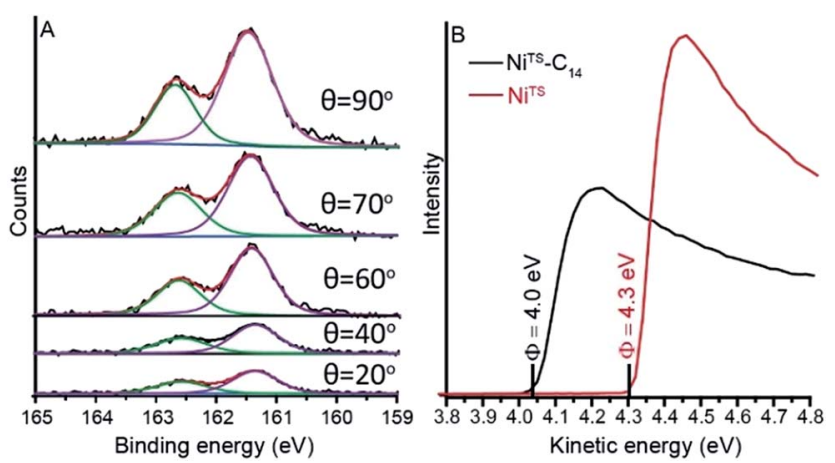

Fig. 4 (A) XPS of S $2 p$ as a function of the take-off angle of $\mathrm{C}_{14} \mathrm{Ni}^{\mathrm{TS}}-$ $\mathrm{SAM}$ and (B) UPS spectra of bare $\mathrm{Ni}^{\mathrm{TS}}$ and $\mathrm{S} \mathrm{SC}_{14}$ SAM coated $\mathrm{Ni}^{\mathrm{TS}}$. the $\mathrm{SC}_{14} \mathrm{SAMs}$ is $1.74 \pm 0.2 \mathrm{~nm}$ in agreement with previous reports considering a molecular length of $2.17 \mathrm{~nm}$ (estimated by CPK model) and a tilt angle with respect to the surface normal of $36 \pm 9^{\circ}$. $^{\mathbf{1 3}, 34,36}$

We determined the work function $\Phi$ of the $\mathrm{Ni}^{\mathrm{TS}}$-SAM and a freshly prepare native $\mathrm{Ni}^{\mathrm{TS}}$ substrate by ultraviolet photoemission spectroscopy (UPS). We found that the adsorbed monolayer caused a lowering of $\Phi$ with respect to the bare $\mathrm{Ni}^{\mathrm{TS}}$ surface of $0.3 \mathrm{eV}$ (Fig. 4B) resulting in a similar value of $\Phi$ as of alkanethiolate SAMs on gold and silver likely due to Fermi-level pinning caused by the Ni-S bond. ${ }^{50-52}$

\section{Stability of Si-FOTS/Ni and $\mathbf{N i}^{\text {TS }}$-SAM}

In principle, since the $\mathrm{Ni}$ is in contact with the template, nickel oxides cannot form. Hence, prior to template-stripping the $\mathrm{Ni}$ surfaces can be stored for months without forming NiO. To test this hypothesis, we measured XPS spectra of O 1s (Fig. 5) from a $\mathrm{Ni}^{\mathrm{TS}}$-SAM surface that was stripped off the Si-FOTS/Ni template after aging of the Si-FOTS/Ni stack for six to eight months in ambient conditions. The $\mathrm{O} 1 \mathrm{~s}$ peak was not observed for $\mathrm{Ni}^{\mathrm{TS}}$-SAM prepared on Si-FOTS/Ni aged up to six months. The $\mathrm{Ni}^{\mathrm{TS}}$-SAM formed on an 8 month old surface showed an $\mathrm{O}$ $1 \mathrm{~s}$ peak at $529.5 \mathrm{eV}$ which could be due to physisorbed $\mathrm{C}-\mathrm{O}$ contaminations or NiO. From this observation, in line with observations made by others for $\mathrm{Ag}^{\mathrm{TS}}$ surfaces ${ }^{39}$ we conclude that the Ni surfaces can be stored for prolonged periods of time and can be stripped of the template on demand to yield oxide free surfaces in ordinary laboratory conditions.

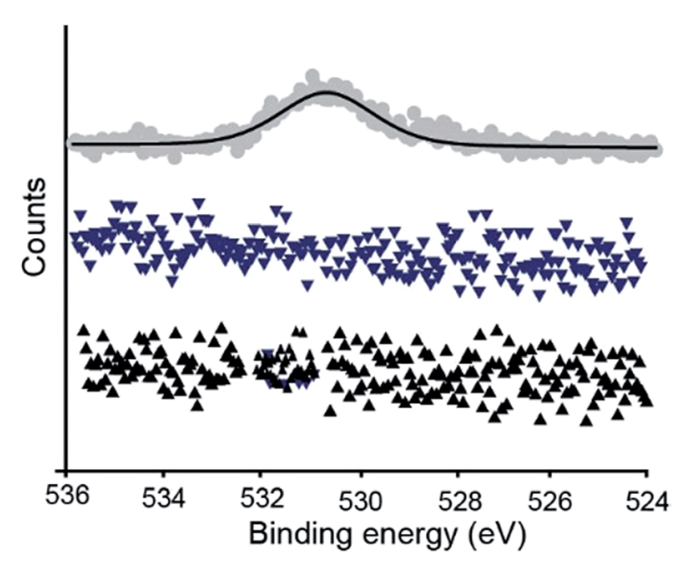

Fig. 5 XPS spectra of $\mathrm{O}$ 1s of $\mathrm{Ni}^{\mathrm{TS}}-\mathrm{SAM}$ substrate with the $\mathrm{Ni}^{\mathrm{TS}}$ template stripped after storing for $(\boldsymbol{\Delta})$ one month, $(\boldsymbol{\nabla}) 6$ months and (-) 8 months of the Si-FOTS/Ni stack. 
The stability of the $\mathrm{Ni}^{\mathrm{TS}}$-SAM surface upon exposure to atmospheric conditions is important to know and so we followed the rate of oxide formation on the $\mathrm{Ni}^{\mathrm{TS}}$-SAM over a period of time of 2 days under atmospheric conditions. Fig. 6A shows that after $1 \mathrm{~h}$ or 1 day of aging no noticeable amounts of oxides were formed. The ratio of the $\mathrm{S} 2 \mathrm{p}$ peak at $162.6 \mathrm{eV}$ and $161.4 \mathrm{eV}$ is $\sim 2: 1$ for $\mathrm{Ni}^{\mathrm{TS}}$-SAM surface exposed in ambient conditions for 1 hour before transfer into the XPS chamber, the observed ratio is consistent with a metal-bound thiolate species (Fig. 6C). Aging for 2 days, however, caused significant degradation of the sample. The O 1s spectrum showed a peak at $529.8 \mathrm{eV}$ and $530.8 \mathrm{eV}$ corresponding to carbon contamination and $\mathrm{Ni}-\mathrm{O}$ indicating that Ni surface started to oxidize and the typical signature of a metal-thiolate bond disappeared. We note that the carbon contamination increased with time as is evident from the decrease in the ratio of the two C 1s signals at 284 (C from the $\mathrm{SAM}$ ) and $285 \mathrm{eV}$ (C from contamination) from 2.4 to 1.1. These results show that the $\mathrm{Ni}^{\mathrm{TS}}$-SAM surfaces are stable for 1 day.

\section{Charge transport studies}

To prove that the $\mathrm{Ni}^{\mathrm{TS}}$ surfaces are suitable for applications in molecular electronics, we formed molecular junctions by contacting the $\mathrm{Ni}^{\mathrm{TS}}$-SAM with cone-shaped EGaIn top-electrodes using well-established procedures. ${ }^{44}$ Fig. 7A shows the $\mathrm{Ni}^{\mathrm{TS}}$ $\mathrm{SC}_{n} / / \mathrm{GaO}_{x} / \mathrm{EGaIn}$ junction schematically. To determine $\beta$ of the $\mathrm{Ni}^{\mathrm{TS}}$-SAM junctions, we prepared SAMs of $\mathrm{SC}_{n}$ (with $n=10,12$, 14, 16 and 18) immobilized on $\mathrm{Ni}^{\mathrm{TS}}$ bottom-electrodes (see ESI $\dagger$ and Table 2). We measured the electrical properties of the $\mathrm{Ni}^{\mathrm{TS}}$ SAM junctions as a function of $n$ (see Experimental section for details) following previously reported procedures. ${ }^{22}$

Fig. 7B shows the log-average $J(V)$ curves for each type of junction. We determined the value of $\beta$ by fitting the data of $\log |J|$ vs. $n$ at $-0.5 \mathrm{~V}$ (Fig. 7C) to the general tunneling equation
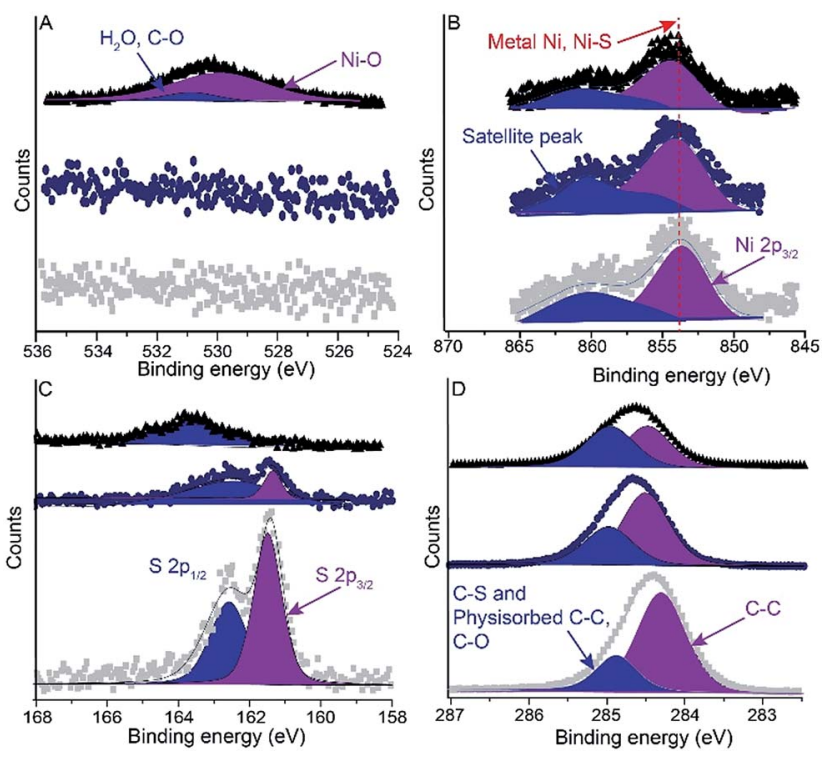

Fig. 6 XPS spectra of $\mathrm{O} 1 \mathrm{~s}(\mathrm{~A}), \mathrm{Ni} 2 \mathrm{p}(\mathrm{B}), \mathrm{S} 2 \mathrm{p}(\mathrm{C})$ and $\mathrm{C} 1 \mathrm{~s}(\mathrm{D})$ after aging in ambient conditions of ( $) \mathrm{Ni}^{\mathrm{TS}}-\mathrm{C}_{14}(1 \mathrm{~h}),(\mathbf{O}) \mathrm{Ni}^{\mathrm{TS}}-\mathrm{C}_{14}$ (1 day) and (4) $\mathrm{Ni}^{\mathrm{TS}}-\mathrm{C}_{14}$ (2 days).

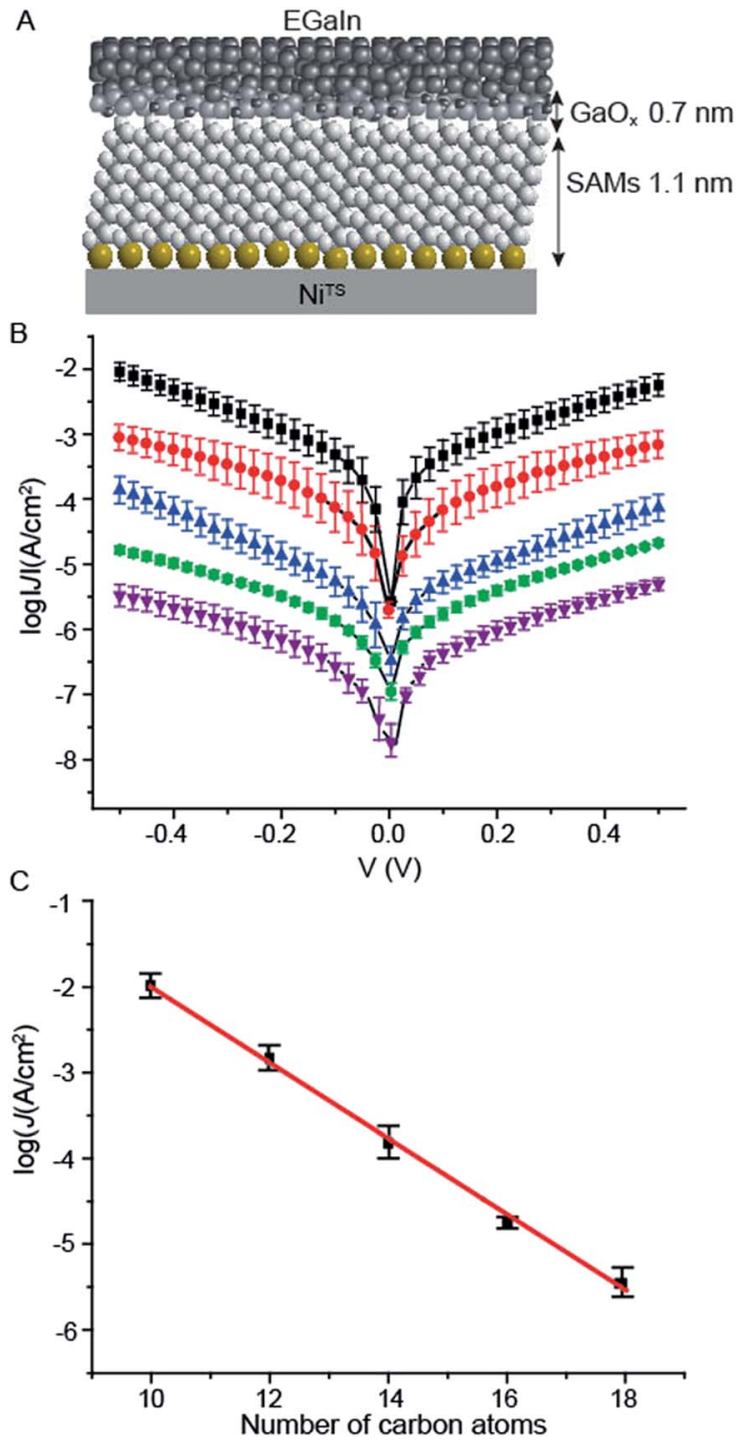

Fig. 7 (A) Schematic of the $\mathrm{Ni}{ }^{\mathrm{TS}}-\mathrm{SAM} / / \mathrm{GaO}_{x} /$ EGaln junctions, (B) plot of the log-average value $J$ vs. $V$ of junctions with a SAM of $(\boldsymbol{\square}) C_{10},(\mathbf{O})$ $\mathrm{C}_{12},(\boldsymbol{\Delta}) \mathrm{C}_{14},(\boldsymbol{\nabla}) \mathrm{C}_{16}$ and $(\boldsymbol{\nabla}) \mathrm{C}_{18}$ and $(\mathrm{C}) \log |\mathrm{J}|$ vs. carbon chain length at $-0.5 \mathrm{~V}$. All error bars represent $95 \%$ confident intervals.

(eqn (1)). The obtained values of $\beta=1.01 \pm 0.03 \mathrm{nC}^{-1}$ and $\log _{10}\left|J_{0}\right|=2.3 \pm 0.2 \mathrm{~A} \mathrm{~cm}^{-2}$ are similar to those values obtained from junctions $\mathrm{Au}^{\mathrm{TS}}$ or $\mathrm{Ag}^{\mathrm{TS}}$ bottom-electrodes. ${ }^{22,46}$ These results indicate that the dominant mechanism of charge transport across our junctions is through-bond tunneling, ${ }^{22,47,53,54}$ where the current flows through the backbone of the alkyl chains of the SAMs. This explains why the values of $J$ of a given molecular length of the SAM are independent of the molecular tilt angles $\alpha$. In other words, the values of $J$ across junctions with alkanethiolate SAMs on $\mathrm{Ni}^{\mathrm{TS}}(\alpha$ $\left.=\sim 39^{\circ} \pm 9^{\circ}\right)$ are the same as those with SAMs on $\mathrm{Ag}^{\mathrm{TS}}(\alpha=$ $\left.\sim 10^{\circ}\right)$ or $\mathrm{Au}^{\mathrm{TS}}\left(\alpha=\sim 30^{\circ}\right)$ substrates. ${ }^{52,53}$ These results indicate that the overall mechanism of charge transport is dominated by through-bond tunneling, and not through space, which is plausible since through-bond tunneling $\left(\beta=0.8 \AA^{-1}\right)$ is much more efficient than through space tunnelling $\left(\beta=2.9 \AA^{-1}\right) .{ }^{20}$ 
Table 2 Statistical analysis of $\mathrm{J}(\mathrm{V})$ measurements of $\mathrm{Ni}^{\mathrm{TS}}-\mathrm{SAM} / / \mathrm{GaO}_{x} /$ EGaln junctions

\begin{tabular}{lllccr}
\hline SAMs & $\begin{array}{l}\text { No. of total } \\
\text { junctions }\end{array}$ & $\begin{array}{l}\text { No. of } \\
\text { shorts }^{a}\end{array}$ & Yield $^{b}$ & $\begin{array}{l}\text { No. of } \\
\text { stable trace }\end{array}$ & $\sigma_{\log }{ }^{c}$ \\
\hline $\mathrm{C}_{10} \mathrm{SH}$ & 17 & 2 & 88 & 375 & 0.44 \\
$\mathrm{C}_{12} \mathrm{SH}$ & 17 & 0 & 100 & 425 & 0.45 \\
$\mathrm{C}_{14} \mathrm{SH}$ & 17 & 1 & 94 & 400 & 0.50 \\
$\mathrm{C}_{16} \mathrm{SH}$ & 14 & 1 & 93 & 325 & 0.34 \\
$\mathrm{C}_{18} \mathrm{SH}$ & 18 & 2 & 89 & 400 & 0.47 \\
Total & 83 & 6 & 92 & 1925 & -
\end{tabular}

${ }^{a}$ A short junction was defined when the value of $J$ exceeded the compliance value of $J$ of our instrument $(100 \mathrm{~mA})$ during data collection. ${ }^{b}$ The yield is defined as the number of non-shorting junctions divided by the total number of junctions. ${ }^{c}$ The $\sigma_{\log }$ is $\log$ standard deviation obtained from Gaussian fitting.

Our previous studies showed that the value of $\beta$ decreased from $1.0 \mathrm{nC}^{-1}$ to $0.4 \mathrm{nC}^{-1}$ with increasing value of $\mathrm{BV}$ from $\sim 10^{5} \mathrm{~nm}^{3}$ to $10^{7} \mathrm{~nm}^{3} .^{39}$ Thus, here the low BV of the $\mathrm{Ni}^{\mathrm{TS}}$ surfaces $(\sim 2 \times$ $10^{5} \mathrm{~nm}^{3}$ ) ensured high quality junctions where the SAMs are good tunneling barriers. These junctions have an average yield of non-shorting junctions of $92 \%$ and good precision of data with log-standard deviations around 0.3-0.5, similar to the same junctions but with $\mathrm{Au}^{\mathrm{TS}}$ and $\mathrm{Ag}^{\mathrm{TS}}$ electrodes.

\section{Conclusions}

We report a procedure for direct self-assembly of $n$-alkanethiolate SAMs on oxide-free (within the detection limits of our XPS system) $\mathrm{Ni}^{\mathrm{TS}}$ substrates without using UHV, glove box, or electrochemical reduction methods. The topography of the $\mathrm{Ni}^{\mathrm{TS}}$ surface was determined by AFM and showed that the surfaces were ultra-flat and that the amount of exposed grain boundaries was small. We also showed these surfaces support densely packed SAMs of $n$-alkanethiolates which, in turn, stabilized the $\mathrm{Ni}^{\mathrm{TS}}$ surface against oxidation in ambient conditions for 1 day. To demonstrate potential applications in molecular electronics, we incorporated the $\mathrm{Ni}^{\mathrm{TS}}$ surface in $\mathrm{Ni}^{\mathrm{TS}}-\mathrm{SAM} / / \mathrm{GaO}_{x} / \mathrm{EGaIn}$ junctions and measured the $J(V)$ characteristics. These measurements showed that the SAMs formed high quality tunnelling barriers and the junctions did not suffer from pin holes, with a high yield of non-shorting junctions with good reproducibility. Currently, we are investigating the $\mathrm{Ni}^{\mathrm{TS}}$ surfaces in tunnelling junctions with magnetically active monolayers.

\section{Acknowledgements}

Prime Minister's Office, Singapore under its Medium sized centre program is also acknowledged for supporting this research. We also acknowledge the Ministry of Education (MOE) for supporting this research under award No. MOE2015-T2-2-134. The authors thank Dr Xiao-Jiang Yu for help at the Singapore Synchrotron Light Source (SSLS) under NUS core support C-380003-003-001.

\section{References}

1 C. Barraud, P. Seneor, R. Mattana, S. Fusil, K. Bouzehouane, C. Deranlot, P. Graziosi, L. Hueso, I. Bergenti, V. Dediu, F. Petroff and A. Fert, Nat. Phys., 2010, 6, 615-620.

2 L. Schulz, L. Nuccio, M. Willis, P. Desai, P. Shakya, T. Kreouzis, V. K. Malik, C. Bernhard, F. L. Pratt, N. A. Morley, A. Suter, G. J. Nieuwenhuys, T. Prokscha, E. Morenzoni, W. P. Gillin and A. J. Drew, Nat. Mater., 2011, 10, 252.

3 P. Campiglio, R. Breitwieser, V. Repain, S. Guitteny, C. Chacon, A. Bellec, J. Lagoute, Y. Girard, S. Rousset, A. Sassella, M. Imam and S. Narasimhan, New J. Phys., 2015, 17, 063022.

4 T. Sergio, G. Marta, D. Sophie, B. Clément, B. Karim, C. Sophie, D. Cyrile, J. Eric, S. Pierre, M. Richard and P. Frédéric, J. Phys.: Condens. Matter, 2016, 28, 094010.

5 P. Tyagi, E. Friebe and C. Baker, J. Nanopart. Res., 2015, 17, 452.

6 H. Einati, D. Mishra, N. Friedman, M. Sheves and R. Naaman, Nano Lett., 2015, 15, 1052-1056.

7 I. Carmeli, K. Senthil Kumar, O. Heifler, C. Carmeli and R. Naaman, Angew. Chem., Int. Ed., 2014, 53, 8953-8958.

8 R. Naaman and D. H. Waldeck, Annu. Rev. Phys. Chem., 2015, 66, 263-281.

9 E. A. Weiss, G. K. Kaufman, J. K. Kriebel, Z. Li, R. Schalek and G. M. Whitesides, Langmuir, 2007, 23, 9686-9694.

10 D. Yoo, T. W. Johnson, S. Cherukulappurath, D. J. Norris and S.-H. Oh, ACS Nano, 2015, 9, 10647-10654.

11 L. Jiang, T. Wang and C. A. Nijhuis, Thin Solid Films, 2015, 593, 26-39.

12 S. Kumar, T. W. Johnson, C. K. Wood, T. Qu, N. J. Wittenberg, L. M. Otto, J. Shaver, N. J. Long, R. H. Victora, J. B. Edel and S. H. Oh, ACS Appl. Mater. Interfaces, 2016, 8, 9319-9326.

13 A. D. Vogt, T. Han and T. P. Beebe, Langmuir, 1997, 13, 33973403.

14 P. G. Hoertz, J. R. Niskala, P. Dai, H. T. Black and W. You, J. Am. Chem. Soc., 2008, 130, 9763-9772.

15 S. Devillers, A. Hennart, J. Delhalle and Z. Mekhalif, Langmuir, 2011, 27, 14849-14860.

16 J. R. Niskala and W. You, J. Am. Chem. Soc., 2009, 131, 1320213203.

17 C. A. Nijhuis, W. F. Reus and G. M. Whitesides, J. Am. Chem. Soc., 2009, 131, 17814-17827.

18 F. C. Simeone, H. J. Yoon, M. M. Thuo, J. R. Barber, B. Smith and G. M. Whitesides, J. Am. Chem. Soc., 2013, 135, 1813118144.

19 H. B. Akkermann and B. D. Boer, J. Phys.: Condens. Matter, 2008, 20, 013001.

20 R. L. McCreery, Chem. Mater., 2004, 16, 4477-4496.

21 K. S. Kumar, R. R. Pasula, S. Lim and C. A. Nijhuis, Adv. Mater., 2016, 28, 1824-1830.

22 L. Jiang, C. S. S. Sangeeth, A. Wan, A. Vilan and C. A. Nijhuis, J. Phys. Chem. C, 2015, 119, 960-969. 
23 W. F. Reus, C. A. Nijhuis, J. R. Barber, M. M. Thuo, S. Tricard and G. M. Whitesides, J. Phys. Chem. C, 2012, 116, 6714-6733.

24 C. Vericat, M. E. Vela, G. Corthey, E. Pensa, E. Cortés, M. H. Fonticelli, F. Ibañez, G. E. Benitez, P. Carro and R. C. Salvarezza, RSC Adv., 2014, 4, 27730.

25 S. Pookpanratana, L. K. Lydecker, C. A. Richter and C. A. Hacker, J. Phys. Chem. C, 2015, 119, 6687-6695.

26 M. Mattera, R. Torres-Cavanillas, J. P. Prieto-Ruiz, H. PrimaGarcia, S. Tatay, A. Forment-Aliaga and E. Coronado, Langmuir, 2015, 31, 5311-5318.

27 S. Tatay, C. Barraud, M. Galbiati, P. Seneor, R. Mattana, K. Bouzehouane, C. Deranlot, E. Jacquet, A. FormentAliaga, P. Jegou, A. Fert and F. Petroff, ACS Nano, 2012, 6, 8753-8757.

28 M. Galbiati, S. Tatay, S. Delprat, C. Barraud, V. Cros, E. Jacquet, F. Coloma, F. Choueikani, E. Otero, P. Ohresser, N. Haag, M. Cinchetti, M. Aeschlimann, P. Seneor, R. Mattana and F. Petroff, Appl. Surf. Sci., 2015, 353, 24-28.

29 R. Han, F. Blobner, J. Bauer, D. A. Duncan, J. V. Barth, P. Feulner and F. Allegretti, Chem. Commun., 2016, 52, 9805-9808.

30 O. Ozatay, P. G. Gowtham, K. W. Tan, J. C. Read, K. A. Mkhoyan, M. G. Thomas, G. D. Fuchs, P. M. Braganca, E. M. Ryan, K. V. Thadani, J. Silcox, D. C. Ralph and R. A. Buhrman, Nat. Mater., 2008, 7, 567-573.

31 L. De Los Santos Valladares, A. Ionescu, S. Holmes, C. H. W. Barnes, A. Bustamante Domínguez, O. Avalos Quispe, J. C. González, S. Milana, M. Barbone, A. C. Ferrari, H. Ramos and Y. Majima, J. Vac. Sci. Technol., $B, 2014$, 32, 051808.

32 T. Y. Chung and S. Y. Hsu, J. Phys.: Conf. Ser., 2009, 150, 042063.

33 S. R. Catarelli, S. J. Higgins, W. Schwarzacher, B. W. Mao, J. W. Yan and R. J. Nichols, Langmuir, 2014, 30, 14329-14336.

34 S. Bengió, M. Fonticelli, G. Benítez, A. H. Creus, P. Carro, H. Ascolani, G. Zampieri, B. Blum and R. C. Salvarezza, J. Phys. Chem. B, 2005, 109, 23450-23460.

35 C. Fontanesi, F. Tassinari, F. Parenti, H. Cohen, P. C. Mondal, V. Kiran, A. Giglia, L. Pasquali and R. Naaman, Langmuir, 2015, 31, 3546-3552.
36 F. Blobner, P. N. Abufager, R. Han, J. Bauer, D. A. Duncan, R. J. Maurer, K. Reuter, P. Feulner and F. Allegretti, J. Phys. Chem. C, 2015, 119, 15455-15468.

37 S. Mukhopadhyay and I. Das, Phys. Rev. Lett., 2006, 96, 026601.

38 X. Chen and R. H. Victora, Appl. Phys. Lett., 2007, 91, 212104. 39 L. Yuan, L. Jiang, B. Zhang and C. A. Nijhuis, Angew. Chem., Int. Ed., 2014, 53, 3377-3381.

40 C. W. Miller, Z.-P. Li, J. Åkerman and I. K. Schuller, Appl. Phys. Lett., 2007, 90, 043513.

41 Z. S. Zhang, J. Appl. Phys., 2004, 95, 557.

42 R. H. Victora and X. Chen, IEEE Trans. Magn., 2010, 46, 702708.

43 Z. Mekhalif, F. Laffineur, N. Couturier and J. Delhalle, Langmuir, 2003, 19, 637-645.

44 L. Yuan, N. Nerngchamnong, L. Cao, H. Hamoudi, E. del Barco, M. Roemer, R. Sriramula, D. Thompson and C. A. Nijhuis, Nat. Commun., 2015, 6, 6324-6335.

45 C. H. Lee, J. H. Kim, C. Zou, I. S. Cho, J. M. Weisse, W. Nemeth, Q. Wang, A. C. van Duin, T. S. Kim and X. Zheng, Sci. Rep., 2013, 3, 2917.

46 R. C. Chiechi, E. A. Weiss, M. D. Dickey and G. M. Whitesides, Angew. Chem., Int. Ed., 2008, 47, 142144.

47 L. Yuan, L. Jiang, D. Thompson and C. A. Nijhuis, J. Am. Chem. Soc., 2014, 136, 6554-6557.

48 S. Frey, K. Heister, M. Zharnikov and M. Grunze, Phys. Chem. Chem. Phys., 2000, 2, 1979-1987.

49 O. Zenasni, A. C. Jamison and T. R. Lee, Soft Mater, 2013, 9, 6356-6370.

50 A. S. Erickson, A. Zohar and D. Cahen, Adv. Energy Mater., 2014, 4, 1301724.

51 B. Kim, S. H. Choi, X. Y. Zhu and C. D. Frisbie, J. Am. Chem. Soc., 2011, 133, 19864-19877.

52 Y. Qi, O. Yaffe, E. Tirosh, A. Vilan, D. Cahen and A. Kahn, Chem. Phys. Lett., 2011, 511, 344-347.

53 H. Song, H. Lee and T. Lee, J. Am. Chem. Soc., 2007, 129, 3806-3807.

54 K. Slowinski, R. V. Chamberlain, C. J. Miller and M. Majda, J. Am. Chem. Soc., 1997, 119, 11910-11919. 